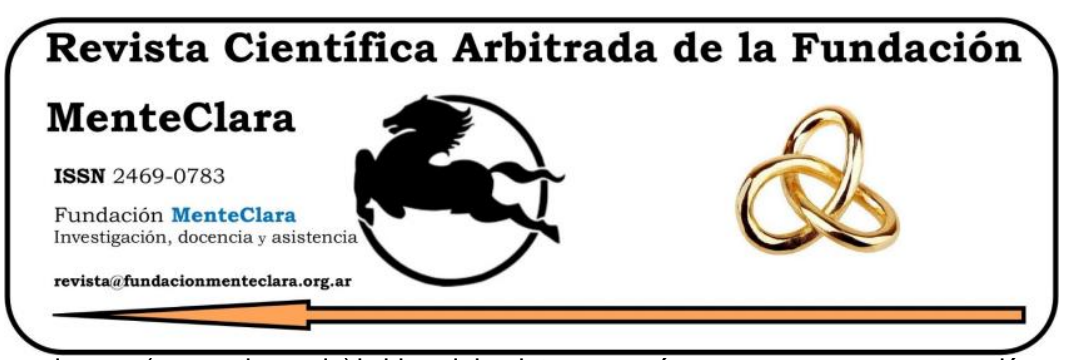

Artículos atravesados por (o cuestionando) la idea del sujeto -y su género- como una construcción psicobiológica de la cultura. Articles driven by (or questioning) the idea of the subject -and their gender- as a cultural psychobiological construction.

Vol. 6 (2021), enero-diciembre ISSN 2469-0783

https: / / datahub.io/dataset/2021-6-e249

\title{
EDUCACIÓN INCLUSIVA Y ACCESIBILIDAD DIGITAL
}

\section{INCLUSIVE EDUCATION AND DIGITAL ACCESSIBILITY}

Selediana de Souza Godinho ${ }^{1}$, Carolina Vivianan Rivela ${ }^{2}$, Suzaneide Oliveira Medrado $^{3}$, Julieta Marmo ${ }^{4}$, Alejandro Lanuque ${ }^{5}$

Cómo citar este artículo / Citation: De Souza Godinho, S.; Rivela, C.V., Medrado, S. O.; Marmo, J.; Lanuque, A. (2021). Educación inclusiva y accesibilidad digital. Revista Científica Arbitrada de la Fundación MenteClara, Vol. 6 (249). DOI: https://doi.org/ 10.32351/rca.v6.249

Copyright: (C) 2021 RCAFMC. Este artículo de acceso abierto es distribuido bajo los términos de la licencia Creative Commons Attribution 4.0 International License (CC BY 4.0). Recibido: 24/08/2021. Aceptado: 04/09/2021 Publicación online: 05/09/2021

Conflicto de intereses: Ninguno que declarar.

\section{Resumen}

El objetivo de este trabajo es analizar el rol que desempeña la accesibilidad digital en la educación inclusiva como un medio de eliminar las barreras frente a múltiples demandas de las personas con discapacidad y los desafios en un entorno de aprendizaje virtual. A través de una revisión sistemática a partir del uso del método PRISMA, se tomaron a 16 artículos que demuestran que la accesibilidad digital en el proceso de inclusión educativa se expresa en una alfabetización y participación colaborativa entre docentes y alumnos con/sin discapacidad, donde las herramientas de TICs deben ser utilizada para favorecen a una igualdad de derechos en el proceso de enseñanza-aprendizaje.

\footnotetext{
1 Universidad de Flores, Argentina. selediana.godinho@uflounivesidad.edu.ar

2 Universidad de Flores, Argentina. carolina.rivela@uflouniversidad.edu.ar

3 Secretaría de la Educación del estado de Bahía, Brasil. suzymedrano@gmail.com

4 Universidad de Flores, Argentina.julietamarmo@yahoo.com.ar

5 Universidad de Flores, Argentina. alejandro.lanuque@uflouniversidad.edu.ar
} 


\begin{abstract}
This study aims to analyze the role that digital accessibility plays in inclusive education as a means of overcoming the barriers given the multiple demands of people with disabilities and the challenges of the virtual learning environment. Through a systematic review based on the usage of the PRISMA method, 16 articles were selected to show that digital accessibility in the inclusive education process is expressed in literacy and the collaboration between teachers and students with and without disabilities, where the ICT tools should be used to promote equality in the teachinglearning process.
\end{abstract}

Palabras Claves: Acce sibilidad Digital; Educación Inclusiva; Tecnología de la Información y la Comunicación

Keyw ords: Accessibility; Inclusive Education; Information and Communications Technology 


\section{Introducción}

La Educación Inclusiva y la Accesibilidad Digital son dos temas que cada vez ganan más relevancia en la sociedad. Por un lado, se percibe la educación como un proceso de inclusión social, a partir de una igualdad de condiciones. Por otro, se aborda la tecnología de la información y la comunicación (TIC) como un medio irreversible que trae consigo nuevas exigencias y oportunidades de enseñanza y aprendizaje en contextos diversos.

Mientras tanto, no se puede dejar de subrayar que la innovación tecnológica es un gran desafio teniendo en cuenta que todavía las personas con discapacidad encuentran diferentes resistencias dentro del proceso de enseñanza tradicional. Además, la pandemia de Covid-19 en el mundo y particularmente en Argentina, aumentó la brecha digital en relación con las medidas adoptadas en la Educación, una vez que los enfoques de aprendizaje se concentraron en el sistema a distancia y en línea con el propósito de continuidad de las actividades didácticas.

Según la Convención sobre los Derechos de las Personas con Discapacidad (ONU, 2006) la educación inclusiva es parte de un proceso de politicas públicas y de prácticas educativas, asentada sobre un derecho humano básico de todas las personas. Además, la Agenda 2030 de las Naciones Unidas (ONU, 2015) describe 17 Objetivos de Desarrollo Sostenible (ODS), presenta en su 4.a Objetivo "Construir y adecuar instalaciones educativas que tengan en cuenta las necesidades de los niños y las personas con discapacidad y las diferencias de género, y que ofrezcan entornos de aprendizaje seguros, no violentos, inclusivos $y$ eficaces para todos".

Se describe un aparato normativo y social de una educación que pone de manifiesto la necesidad de hacer realidad diferentes procesos y 
situaciones para una inclusión social. Mientras tanto, en Argentina y como bien plantea Cinquegrani (2020), la educación inclusiva se presenta como una deuda de un sistema educativo que resiste a los estudiantes con discapacidad. La autora sigue explicando que todavía prevalece la necesidad de acomodar a las personas con discapacidad a los parámetros escolares preestablecidos, exigiendo que los alumnos cumplan con ciertos modelos y procesos que generan situaciones de abierta exclusión (Cinquegrani, 2020).

Mientras tanto, se observa un aumento de las demandas de la educación inclusiva y que requiere de cambios significativos en el entorno educativo. Dentro de este marco es que aparece el tema de la accesibilidad digital, donde se observa el imperativo de proporcionar tecnologias que ayuden a eliminar las barreras (física, contenidos, materiales, entre otras) para que las personas con discapacidad, independientemente de sus limitaciones puedan acceder a un aprendizaje continuo (Hamburg y Bucksch, 2017).

En este caso, se trata de poder percibir desde las TICs, las diferentes necesidades de aquellos que ya cargan en su historia social, percepciones y características negativas y que buscan superar distintos estereotipos y las prácticas y políticas discriminatorias (Barton, 2001). No obstante, no se descarta la brecha digital, que va más allá de las desigualdades en el campo de la discapacidad. Ya que, en Argentina muchos de los productos y servicios tecnológicos no son accesibles, así como, presentan carencias que obstaculizan un acceso digital según diferentes demandas educacionales, económicas y sociales, entre otras.

Asimismo, el contexto actual exhorta a la construcción de proyectos e iniciativas que deben incorporar las tecnologias digitales en la Educación. Desde allí, y retomando a Barton (2001) se puede decir, que analizar la accesibilidad digital en la educación inclusiva es problematizar la 
discapacidad desde ciertos puntos que dan vueltas todo el tiempo y requieren de un estudio profundo con referencia a una sociedad pronta a atender las diferentes demandas presentes en la cotidianidad.

Igualmente, el acceso digital induce a repasar los objetivos de aprendizaje capaces de impulsar el proceso de enseñanza y aprendizaje con las personas con discapacidad, a partir de un conjunto de actividades pensadas para el desarrollar de las capacidades personales de los alumnos (Omodei et. al, 2016).

Sin embargo, los obstáculos del acceso digital en las personas con discapacidad pueden ser abordados desde tres aspectos: la asequibilidad, la accesibilidad de los productos y contenidos y la alfabetización digital. En gran parte estas barreras están relacionadas con las condiciones económicas, niveles de estudios, una vez que las personas con mayor acceso a las TICs tienden a tener mayores tasas de poder adquisitivo, escolaridad y status social. Consecuentemente, la accesibilidad digital tiene una correlación directa con las condiciones sociales, económicas y los diferentes niveles de apropiación de la tecnología. (García-Prieto, 2016; Prioste y Raiça, 2017).

Si bien, para muchos el acceso digital parece algo futurístico, no se puede dejar de considerar que la tecnología, está cada vez más presente en diferentes ámbitos, y que sucede a cada momento y pone la discapacidad en un lugar de marginalidad y exclusión social, cuando en las nuevas formas de pensar la Educación, no se concreta el uso de los nuevos modelos de enseñanza con el propósito de la inclusión social (Cabero Almenara y Palmero, 2017).

El tema abre un análisis, no solo desde la calidad que requiere una verdadera educación inclusiva, como por la relevancia que ocupa la tecnología en este escenario y remite a la pregunta: ¿qué papel puede 
jugar la accesibilidad digital en el proceso de enseñanzay aprendizaje de las personas con discapacidad?

Desde allí, este estudio tiene como objetivo general analizar el rol que desempeña la accesibilidad digital en la educación inclusiva como un medio de eliminar las barreras frente a múltiples demandas de las personas con discapacidad y los desafios en un entorno de aprendizaje virtual.

\section{Antecedentes}

Romo (2017) aborda la relación entre las tecnologías de la información y la comunicación y las escuelas de las zonas rurales. El objetivo de la investigación fue verificar si la tecnología contribuye al proceso de educación inclusiva, donde el aislamiento social ya es una característica de las escuelas rurales. De esta manera, lo que se busca saber es si la brecha digital sigue perpetuándose en estos contextos geográficos y sociales. El estudio fue realizado en el entorno rural de Castilla y León, España, con base a una metodología cualitativa de 36 entrevistas personales y 4 grupos focales de padres/madres, docentes, directores/as, representantes de asociaciones de padres/madres (AMPA) y alumnado. Los resultados obtenidos muestran que los alumnos de escuelas rurales hacen un uso mayor de las herramientas tecnológicas que los alumnos urbanos. Sin embargo, los problemas de igualdad de oportunidades siguen iguales a pesar de las importantes mejoras en equipamientos. Todavía persiste la problemática en cuanto a la formación docente, accesibilidad, aceptabilidady adaptabilidad de los entornes de enseñanza y aprendizaje, lo que dificulta el alcance de un concepto de justicia social. 
Cabero-Almenara y o Ruiz-Palmero (2017) también abordan el concepto de "brecha digital" para hacer referencia a la accesibilidad digital en la educación, donde perciben que el desconocimiento sobre el uso de las TICs dificulta el proceso de inclusión y hasta el propio concepto de educación inclusiva, que no debe atarse solamente a las personas con discapacidad, sino a la incorporación de todas las personas que están excluidas del sistema educacional, sea por género, cultura, etnia, situación económica, entre otros aspectos que genera exclusión. En su investigación asumen todavía, que la inclusión y la integración, son dos procesos diferentes. Por un lado, la integración se centra en el alumno, en su evaluación y diagnósticos. Por otro lado, la inclusión, aborda los factores de enseñanza-aprendizaje, a la búsqueda por resolver los problemas, la colaboración y la construcción de estrategias para que los docentes puedan desarrollar un espacio de flexibilidad para la incorporación de los alumnos.

De este modo, la investigación bibliográfica de Cabero-Almenara y o Ruiz-Palmero (2017), pone en enfoque al diseño accesible, con criterios relacionados a los materiales educativos y a los principios éticos centrados en la persona y en generar medios para facilitar su participación en el proceso de aprendizaje.

Favorecer la inclusión con las TIC, es tener presente desde un principio que se debe orientar su selección al docente, es el de ofrecer al estudiante una diversidad de códigos y sistemas simbólicos para favorecer de esta forma su mayor adecuación a las inteligencias múltiples de los alumnos y a su diversidad de estilos de procesamiento de la información y de aprendizaje (Cabero-Almenara y Ruiz-Palmero, 2017, p.21)

Palomino (2017) describe las percepciones de los futuros docentes de Educación Infantil y Primaria en relación con el uso de las TICs en el desarrollo de prácticas inclusivas en el aula. A través de una encuesta 
con 231 estudiantes del Grado de Educación Infantil y Primaria de la Universidad Católica de Murcia, alcanzó resultados que demuestran percepciones optimistas de los futuros docentes hacia las posibilidades del uso de estos recursos en el proceso de enseñanza-aprendizaje de los alumnos con discapacidad. Además, la encuesta presenta como hallazgos relevantes que las TICs son observadas como un factor clave para la atención a la diversidad, como así posibilitan un proceso de enseñanza-aprendizaje según las demandas personales de los alumnos. Por último, el estudio destaca el use de TICs para la capacitación profesional y formación curricular de los nuevos sistemas didácticos basados en el diseño universal de aprendizaje.

En un análisis sobre la educación inclusivay el acceso digital, Cardoso y Giraffa (2019) perciben un gran desafio en el sistema escolar, principalmente, porque todavía observan una resistencia al desarrollo integral de los estudiantes según sus diversidades. Asimismo, observan que el propio concepto de inclusión escolar implica abarcar diferentes aspectos, tales como, físico, económico, género, tecnológico, entre otros. En su discusión teórica, los autores parten del término "alfabetización digital pedagógica" como uno de los suportes para ascender a las mejores prácticas inclusivas en la educación, no solo desde las estrategias de enseñanza y aprendizaje, con mejoras en los programas de enseñanza, como por la posibilidad de que los alumnos puedan compartir el saber de distintas maneras, por medio de practica innovadoras y dentro de un planeamiento colaborativo entre escuela, familia y alumnos.

Zaduski, et.al. (2019) en una investigación sobre ecosistemas de aprendizaje, describen que el espacio escolar debe ser un ambiente en que todos puedan aprender unos de los otros, en un movimiento de pensamientos divergentes, nuevas ideas y cambios de paradigmas, creencias y convicciones. Desde un abordaje construccionista 
contextualizado y significativo, los autores fomentan la discusión de una educación a distancia en cuanto escenario de encuentro e inclusión. En una investigación acción basado en un trabajo de ámbito internacional y colaborativo, con abordaje CCS, para la construcción de ambientes de aprendizaje on-line alcanza resultados que indican el imperativo de que la escuela acompañe la evaluación tecnológica con posibilidades del uso de Inteligencia Artificial, que permite, por ejemplo, la evaluación personalizada, la seguridad digital y el acceso a la información en diferentes puntos del planeta. Asimismo, en este campo aparece el diseño universal como un instrumento para pensar, planear y crear mejores condiciones de acceso y permanencia de las personas con discapacidad en el ámbito escolar.

En un dosier sobre inclusión digital y accesibilidad educacional enfocado en la diversidad de los alumnos, Pletsch, et.al (2020) presentan algunas posibilidades de una educación inclusiva a través de instrumentos tecnológicos y basados en la tecnología asistiva. El documento presenta resultados de investigaciones del uso de las redes sociales, como Facebook y aplicativos, como WhatsApp, que son utilizados por los docentes para planeamiento pedagógico, durante la Pandemia Covid-19, donde fue necesario buscar soluciones urgentes para disminuir la desigualdad social fomentada por el distanciamiento social de millones de niños, jóvenes y adultos sin el acceso escolar y universitario. Destacan con eso que comprender la tecnología, ya no es solo una mirada de un objeto o recurso pedagógico, sino el elemento cultural para que todos tengan acceso a la educación y justamente es esta concepción de cultura que urge de una demanda para que también las personas con discapacidad no queden alejadas en la producción cibercultural, en que la autonomía de aprendizaje es un hecho social. 
Según, Rocha et. al. (2021) las nuevas tecnologías de la información y comunicación ya son partes de las transformaciones culturales en la contemporaneidad del ámbito escolary presentan diferentes desafíos que rompen con el modelo tradicional de construcción del conocimiento, no pueden pasar desapercibidos cuando el tema es la inclusión. A través de una investigación bibliográfica, abordan sobre los juegos digitales, como un repensar metodológico para la promoción del aprendizaje de los alumnos con discapacidad. Para ello, alcanzan la propuesta de que el alumno que está dentro del sistema regular de enseñanza, también reciba un trato educacional especializado en un aula de recursos multifuncionales, por medio del uso de la tecnología asistiva. Consideran, los autores que la tecnología asistiva, presenta diferentes recursos y herramientas para la autonomía y comunicación de las personas con discapacidad.

\section{Materiales y métodos}

Esta investigación fue realizada con el uso del método PRISMA, según los pasos que certifican una revisión sistemática de calidad y con rigor científico. Por un lado, fueron incluidos los siguientes criterios: el título, identificando la publicación como una revisión sistemática (1); el resumen estructurado (2); la justificación y objetivos, descriptos en la introducción de este estudio (3 y 4); el método, con los criterios de elegibilidad, las fuentes de información, búsqueda, la selección, el proceso de recopilación de datos y las variables para la búsqueda de los datos ( 6 a 11); los resultados, con la selección y características de los estudios (17 y 18); la discusión, en que las evidencias, los hallazgos, limitaciones, conclusiones, colaboración y financiamiento (de 23 a 27). Por otro lado, los criterios de exclusión fueron: protocolo y registro (5); los efectos de justipreciar el riesgo de sesgo en los estudios individuales 
(12); las medidas de resumen (13); los procesos de sintesis de los resultados, procesamientos de datos y combinación de resultados (14); los riesgos de sesgo entre los estudios (15a 18 y 20 a 22); el bien como el riesgo de sesgo en los estudios (19). La revisión sistemática se concentró en la educación inclusiva y el rol de la accesibilidad digital en este contexto, a partir de una exhaustiva búsqueda de artículos publicados sobre esta temática.

\section{Estrategia de búsqueda y selección de los artículos}

La primera búsqueda fue en relación con los artículos indexados a las bases de datos: DOAJ (Directorio de revistas de acceso abierto), REDIB (Red Iberoamericana de Innovación y Conocimiento Científico), Redalyc (Redalyc Red de Revistas Científicas de América Latina y el Caribe, España y Portugal), SCIELO (Scientific Eletronic Library Online), Dialnet, BVS (Biblioteca Virtual en Salud) y PubMed. Se utilizó como descriptores las palabras-clave: Educación Inclusiva, Accesibilidad Digital. La búsqueda fue realizada de forma separada y sin filtros específicos, con el objetivo de medir la cantidad de artículo que versan sobre los temas analizados. En este sentido, se alcanzó un total de 19.004 (Educación Inclusiva - 16.363 y Accesibilidad Digital - 2.641) artículos.

En una segunda búsqueda con la intersección de los términos: Educación Inclusiva y Accesibilidad Digital, se observa una baja significativa en los filtros (28 artículo), como también que algunas plataformas de datos no tenían publicaciones específicas sobre los descriptores utilizados.

A partir de allí, en una tercera cribado y con una evaluación de los artículos y exclusión de 11 publicaciones que no eran de pertinencia 
específica de este estudio y 1 duplicado, se alcanzó 16 artículos a ser revisado. Esta revisión fue realizada entre 02 de febrero y 26 de mayo de 2021. El periodo analizado de las publicaciones fue de 01 de enero de 2016 hasta febrero de 2021.

Diagrama de Flujo

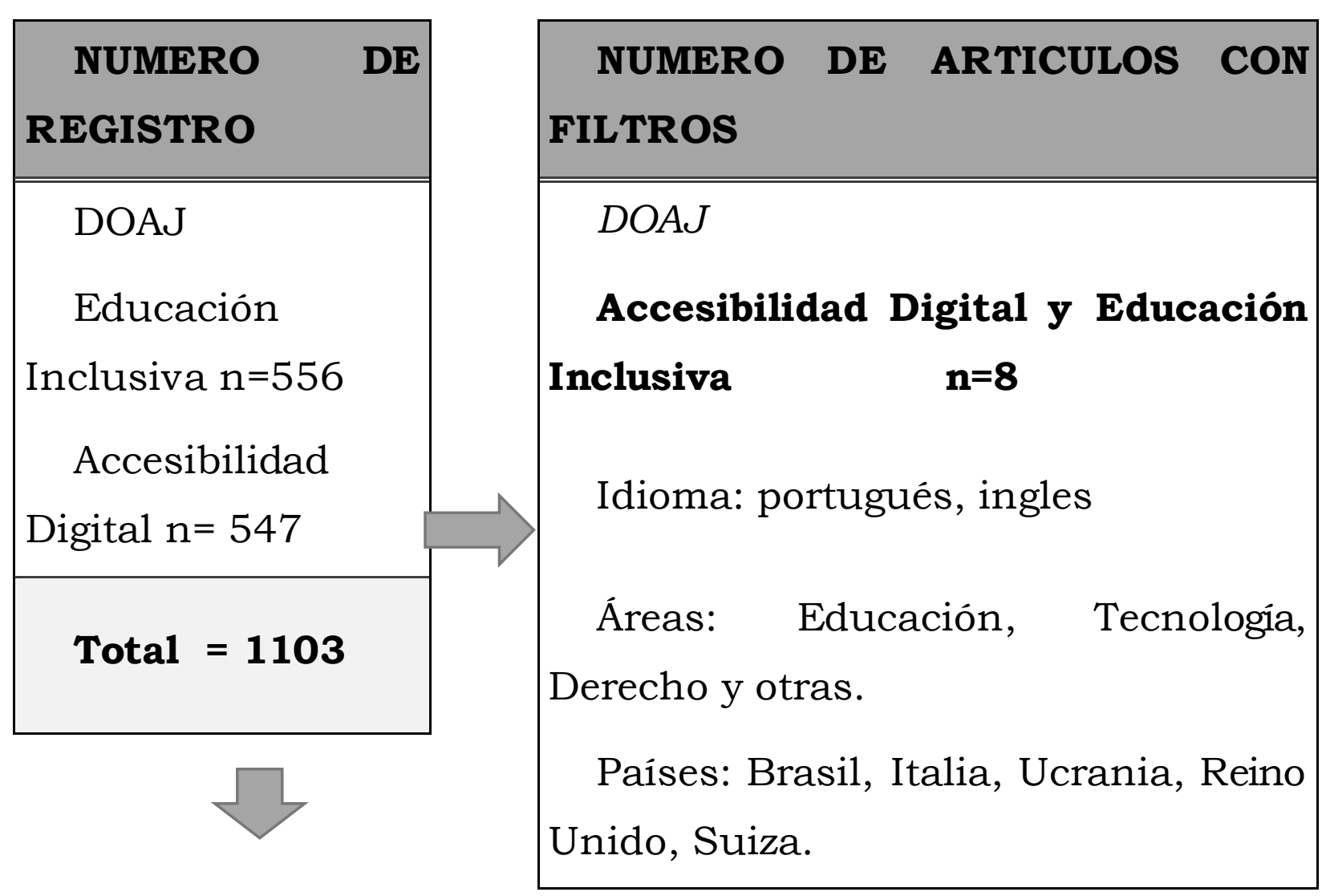




\begin{tabular}{|c|c|}
\hline $\begin{array}{r}\text { NUMERO DE } \\
\text { REGISTRO }\end{array}$ & \\
\hline REDIB & $\begin{array}{l}\text { NUMERO DE ARTICULOS CON } \\
\text { FILTROS }\end{array}$ \\
\hline $\begin{array}{l}\text { Educación } \\
\text { Inclusiva } n=3303 \\
\text { Accesibilidad } \\
\text { Digital } n=580\end{array}$ & $\begin{array}{l}R E D I B \\
\text { Accesibilidad Digital y Educación } \\
\text { Inclusiva } \quad \mathbf{n}=\mathbf{9}\end{array}$ \\
\hline Total $=3883$ & $\begin{array}{l}\text { Idiomas: Español, Portugués e ingles } \\
\text { Áreas: Educación, Tecnologia, } \\
\text { Ciencias Sociales y Humanidades, } \\
\text { Países, Brasil, España, Costa Rica, } \\
\text { Argentina, México, Portugal y otros. }\end{array}$ \\
\hline $\begin{array}{r}\text { NUMERO DE } \\
\text { REGISTRO }\end{array}$ & \\
\hline $\begin{array}{l}\text { REDALYC } \\
\text { Educación } \\
\text { Inclusiva } \mathrm{n}=1677\end{array}$ & \\
\hline $\begin{array}{l}\text { Accesibilidad } \\
\text { Digital } \mathrm{n}=7\end{array}$ & $\begin{array}{l}\text { NUMERO DE ARTICULOS CON } \\
\text { FILTROS }\end{array}$ \\
\hline Total $=1684$ & $\begin{array}{l}\text { SCIELO } \\
\text { Accesibilidad Digital y Educación } \\
\text { Inclusiva } \quad \mathbf{n}=\mathbf{2} \\
\text { Idiomas: portugués }\end{array}$ \\
\hline
\end{tabular}




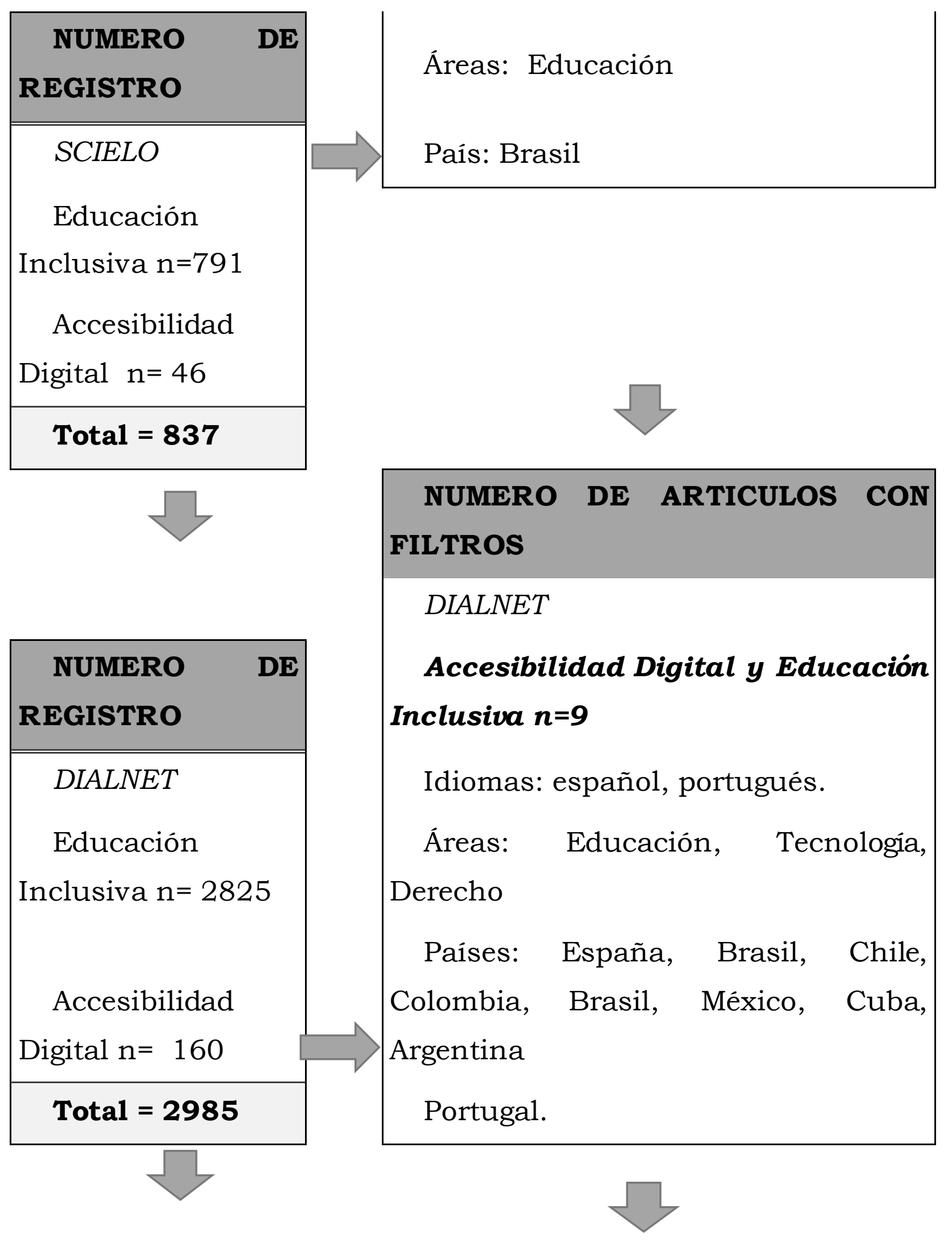




\begin{tabular}{|c|}
\hline NUMERO DE \\
REGISTRO \\
\hline \hline BVS \\
Educación \\
Inclusiva $\mathrm{n}=344$ \\
Accesibilidad \\
Digital $\mathrm{n}=1009$ \\
\hline Total $=\mathbf{1 3 5 3}$ \\
\hline
\end{tabular}

\begin{tabular}{|c|}
\hline NUMERO DE \\
REGISTRO \\
\hline \hline PUBMED \\
Educación \\
Inclusiva $\mathrm{n}=6867$ \\
Accesibilidad \\
Digital $\mathrm{n}=292$ \\
\\
Total $=\mathbf{7 1 5 9}$ \\
\hline
\end{tabular}

\section{EXCLUIDOS}

\begin{tabular}{|c|c|c|}
\hline DOAJ & REDIB & DIALNET \\
\hline SCIELO & & \\
\hline $\mathrm{n}=3$ & $\mathrm{n}=3$ & $n=4$ \\
\hline
\end{tabular}

Total 11

\section{DUPLICADOS}

REDIB/SCIELO

$$
\mathrm{n}=1
$$

TOTAL DE EXCLUIDOS Y DUPLICADOS $=10$ 


\section{$\begin{array}{|cc|}\text { Total } & \text { de } \\ \text { articulos } & =19004\end{array}$}

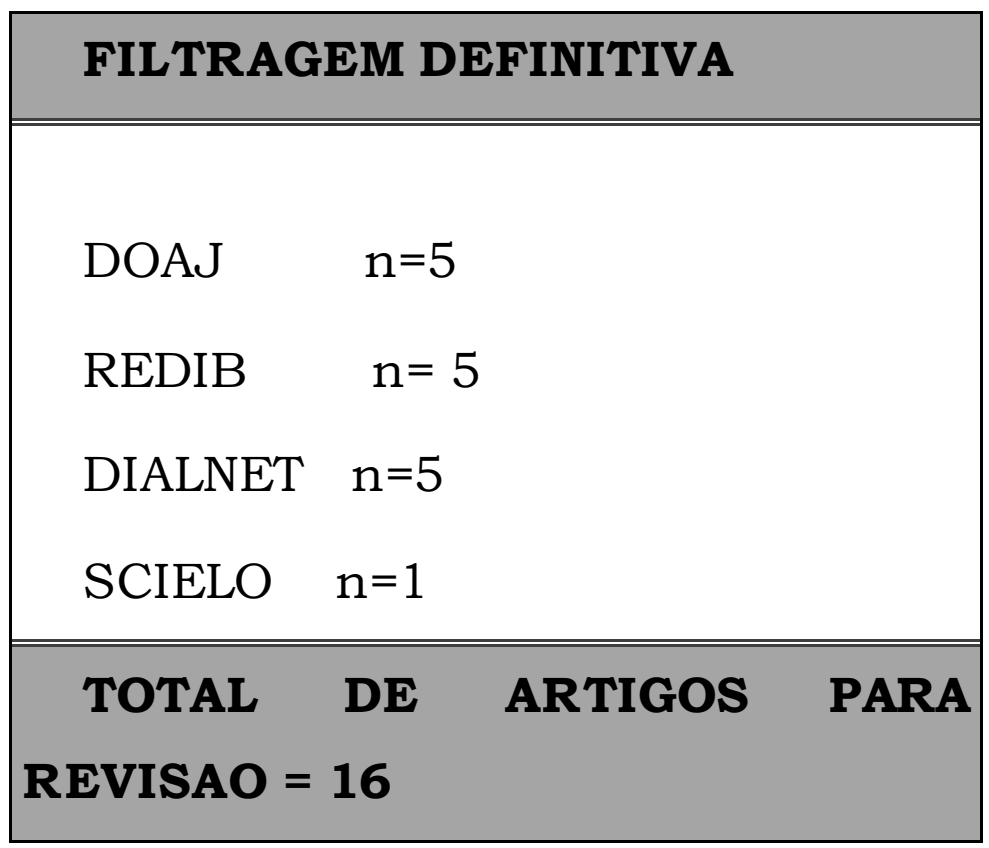

\section{Resultados}

Los artículos seleccionados y analizados en esta investigación abordan los siguientes temas en relación con la accesibilidad digital y educación inclusiva: TICs y educación superior inclusiva $(31,25 \%)$; Tecnología asistiva y discapacidad visual (25\%); Trabajo colaborativo, formación docente y tecnología digital (18,75\%); alfabetización, educación primaria y secundaria, TICs e inclusión (18,75\%); dispositivos móviles, tecnología asistiva y personas con autismo $(6,25 \%)$.

En cuanto a la metodología aplicada, los estudios se concentran en: encuestas $(31,25 \%)$, entrevistas $(18,75 \%)$, estudio de caso $(25 \%)$, revisión sistemática de literatura (25\%). De estos estudios, 62,50\% fueron investigaciones cualitativas, $31,25 \%$ cuantitativas, y 6,25\% mixto.

En relación con la delimitación del objeto de estudio, se observa que el tema central es la inclusión a partir de la tecnologia y herramientas digitales en diferentes contextos, que van desde la alfabetización, a la participación colaborativa entre docentes y alumnos con/sin 
discapacidad y diferentes proyectos que permiten una igualdad y participación en el proceso de enseñanza-aprendizaje.

Se destaca en los artículos los cambios sociales y legales en relación con a la inclusión social en el ámbito educativo (Burci y Çosta, 2018), donde los desafios del aprendizaje inclusivo a distancia son estudiados desde la alfabetización de la educación hasta los trabajos colaborativos, como, por ejemplo, en educación superior en grupos formados entre distintas universidades, como, por ejemplo, The Open University (OU), la Universidad de Leeds y la Universidad de Plymouth Quales con el objetivo de evaluar, ampliar y promover una accesibilidad digital e inclusión de estudiantes con discapacidad (Person, 2019). Desde allí, los estudios abordan la alfabetización digital y mediática, como parte del derecho básico en las sociedades democráticas (Prioste y Raiça, 2017; del Rio et al, 2018).

Es interesante citar los avances en la educación superior inclusiva a distancia especialmente lo que se describe de forma integrada, en que la accesibilidad aparece como un medio de los recursos digitales reutilizables, para que las personas con discapacidad pueden tomar iniciativas en su proceso de aprendizaje, así como para el crecimiento profesional. (Omodei et al, 2016; Zapata, 2016; Person, 2019; Rodriguez et.al., 2020)

De esta manera, la relevancia de las TCIs en relación con el problema de accesibilidad educativa de las personas con discapacidad es parte de una búsqueda constante para mejorar la calidad de los servicios ofertados a las personas con discapacidad (Bruno y Nascimento, 2019; Budny y Kotyk, 2020).

Además, la tecnología aparece como medio de democratización y accesibilidad digital de bajo costo. (Tucci et.al. (2020). La oportunidad de 
recursos y herramientas asistivas, como aplicativos gratuitos desarrollados para potencializar el aprendizaje a distancia, contribuye a una perspectiva de educación inclusiva. (Toledo Morales y Llorente Cejudo, 2016; Silveira et.al, 2020). También construye un entorno virtual y materiales disponibles para las personas con discapacidad, siendo ellos en su mayoría pensados para las barreras de nivel sensorial (Omodei et al, 2016; Medrano y Danta, 2018; Bruno y Nascimento; 2019; Alotaibi et.al, 2020)

Sin embargo, algunas de las investigaciones aclaran que la temática Educación Inclusiva y Accesibilidad Digital, todavía es reciente, mientras tantos, los estudios ya sirven para fundamentar la contribución de la tecnología en el proceso inclusión educativa (Burci y Çosta, 2018; Arias Flores et al., 2017; Silveira et.al, 2020)

En este contexto, emergen indicadores y terminologias que son cada vez más determinantes para un proceso de inclusión digital y social. Son ellas; conectividad, virtualización de los contenidos; accesibilidad, recursos digitales, competencias digitales, educomunicación, entre otras. (Zapata, 2016; Toledo Morales y Llorente Cejudo, 2016; Rojas-Rojas et al. (2017)

También están los desafios en la mediación del docente, en su formación, en la accesibilidad de los ambientes virtuales de aprendizaje y en el dominio del uso de las tecnologías por parte de los profesores y estudiantes. (Burci y Çosta, 2018). Todavía es imperativo la promoción de las tecnologías asistenciales que permitan a los alumnos con discapacidad obtener una educación más efectiva, comunicarse por medio de dispositivos, incluso de manera remota. en referencia a las actitudes y habilidades profesionales (Arias Flores et al., 2017; Budny y Kotyk, 2020; Rojas-Rojas et al., 2017; Rodriguez et al., 2020) 
Se observa que los estudios en parte se concentran en determinadas discapacidades, principalmente en el campo visual, seguido de los estudios de la discapacidad auditiva y para personas con autismo. En consecuencia, otros tipos de discapacidad dejan de ser atendidas (Silveira et.al, 2020; Rojas-Rojas et al., 2017).

Se agrega brechas tecnológicas por la falta de infraestructura y apoyo a los docentes y las dificultades de las personas con discapacidad en la apropiación de los recursos tecnológicos, lo que en ciertos casos imposibilita que los mismo accedan a mejores condiciones de vida y participación social. (Prioste y Raiça; 2017; Burci y Çosta, 2018; RojasRojas et al. 2017)

\begin{tabular}{|c|c|c|c|c|}
\hline $\begin{array}{r}\text { Aut } \\
\text { or (es)/ } \\
\text { Año }\end{array}$ & $\begin{array}{l}\text { M étodo } \\
\text { / Instru } \\
\text { mento }\end{array}$ & Población & Resultados & $\begin{array}{l}\text { Área del } \\
\text { conocimien } \\
\text { to/Base de } \\
\text { Datos }\end{array}$ \\
\hline $\begin{array}{l}\text { Omo } \\
\text { dei et } \\
\text { al } \\
(2016)\end{array}$ & $\begin{array}{l}\text { Bibliográ } \\
\text { fica y } \\
\text { estudio de } \\
\text { caso }\end{array}$ & \begin{tabular}{l}
\multicolumn{1}{c}{ Curso de } \\
Especializaci \\
ón en \\
Educación \\
Especial \\
desde la \\
Perspectiva \\
de la \\
Educación \\
Integrada de \\
la \\
Universidad \\
de San Pablo.
\end{tabular} & $\begin{array}{l}\text { Os resultados } \\
\text { evidenciaron un } \\
\text { esfuerzo por parte de la } \\
\text { universidad en } \\
\text { impulsar la } \\
\text { accesibilidad en todo el } \\
\text { entorno virtual y } \\
\text { materiales disponibles. } \\
\text { mientras tanto, se } \\
\text { observa la necesidad } \\
\text { de adaptaciones en los } \\
\text { objetos de aprendizaje } \\
\text { para que sean } \\
\text { totalmente accesibles a }\end{array}$ & $\begin{array}{l}\text { Educación } \\
\text { a distancia - } \\
\text { REDIB }\end{array}$ \\
\hline
\end{tabular}




\begin{tabular}{|c|c|c|c|c|}
\hline & & & $\begin{array}{l}\text { personas con } \\
\text { discapacidad. }\end{array}$ & \\
\hline $\begin{array}{l}\text { Toledo } \\
\text { Morale } \\
\text { s y } \\
\text { Llorent } \\
\text { e } \\
\text { Cejudo } \\
\text { (2016) }\end{array}$ & $\begin{array}{l}\quad \text { Cuestion } \\
\text { ario } \\
\text { COTETICNE } \\
\text { (Cabero } \\
\text { et.al, 2016) }\end{array}$ & $\begin{array}{l}\quad 154 \\
\text { estudiantes } \\
\text { del Grado de } \\
\text { Educación } \\
\text { Primaria de } \\
\text { la } \\
\text { Universidad } \\
\text { de Sevilla. }\end{array}$ & $\begin{array}{l}\text { Los resultados } \\
\text { indican que los } \\
\text { estudiantes presentan } \\
\text { muy poco } \\
\text { conocimiento respecto } \\
\text { a la aplicación de las } \\
\text { TIC para personas con } \\
\text { algún tipo de } \\
\text { Discapacidad. } \\
\text { Además, este } \\
\text { conocimiento varía } \\
\text { según el tipo de } \\
\text { discapacidad. }\end{array}$ & $\begin{array}{l}\text { Educación } \\
\text { Digital } \\
\text { DIALNET }\end{array}$ \\
\hline $\begin{array}{l}\text { Zap } \\
\text { ata } \\
\text { 6) }\end{array}$ & $\begin{array}{l}\text { Cuantitat } \\
\text { iva de tipo } \\
\text { explicativa } \\
\text { con un } \\
\text { estudio } \\
\text { transeccion } \\
\text { al, encuesta } \\
\text { con escala } \\
\text { de Likert, } \\
\text { estadística } \\
\text { inferencialy } \\
\text { análisis de } \\
\text { varianza } \\
\text { ANOVA y la } \\
\text { prueba Post }\end{array}$ & $\begin{array}{l}\text { Los } \\
\text { sujetos que } \\
\text { participaron } \\
\text { fueron los } \\
\text { directivos de } \\
\text { la UNAD y de } \\
\text { la UPN. }\end{array}$ & $\begin{array}{l}\quad \text { Los resultados } \\
\text { identifican que los } \\
\text { indicadores } \\
\text { Accesibilidad y la } \\
\text { Inclusión digital son } \\
\text { bajos y difieren } \\
\text { significativamente } \\
\text { respecto a sus medias } \\
\text { en universidades } \\
\text { públicas UNAD y UPN. } \\
\text { De este modo, las TICs } \\
\text { no son utilizadas en la } \\
\text { Educación inclusiva. }\end{array}$ & $\begin{array}{l}\text { Educación } \\
\text { Mediática y } \\
\text { TIC - } \\
\text { DIALNET }\end{array}$ \\
\hline
\end{tabular}




\begin{tabular}{|c|c|c|c|c|}
\hline & $\begin{array}{l}\text { Hoc de } \\
\text { Turkey }\end{array}$ & & & \\
\hline $\begin{array}{l}\quad \text { Aria } \\
\text { s } \\
\text { Flores } \\
\text { et.al } \\
(2017)\end{array}$ & $\begin{array}{l}\text { Estudio } \\
\text { de caso }\end{array}$ & $\begin{array}{l}\text { Estudiante } \\
\text { con } \\
\text { discapacidad } \\
\text { visual }\end{array}$ & $\begin{array}{l}\text { El resultado } \\
\text { evidencia la falta de } \\
\text { estrategias pedagógicas } \\
\text { y de prácticas } \\
\text { inclusivas en los } \\
\text { programas de } \\
\text { educación superior, así } \\
\text { como muestra el } \\
\text { imperativo del diseño y } \\
\text { creación de materiales } \\
\text { educativos digitales } \\
\text { accesibles. }\end{array}$ & $\begin{array}{l}\text { Tecnología } \\
- \\
\text { DIALNET }\end{array}$ \\
\hline $\begin{array}{l}\text { Prio } \\
\text { ste y } \\
\text { Raiça } \\
(2017)\end{array}$ & $\begin{array}{l}\text { Cualitativ } \\
\text { a y } \\
\text { exploratoria } \\
\text { con revisión } \\
\text { de } \\
\text { literatura. }\end{array}$ & $\begin{array}{l}\text { Escuelas } \\
\text { públicas } \\
\text { brasileñas. }\end{array}$ & $\begin{array}{l}\text { Los resultados } \\
\text { demuestran que las } \\
\text { escuelas públicas } \\
\text { brasileñas enfrentan } \\
\text { serias brechas en } \\
\text { cuanto la } \\
\text { infraestructura } \\
\text { tecnológica y apoyo a } \\
\text { los docentes para la } \\
\text { inclusión de las } \\
\text { personas con } \\
\text { discapacidad. los } \\
\text { problemas enfrentados } \\
\text { en relación con la } \\
\text { virtualización se } \\
\text { describen en: }\end{array}$ & $\begin{array}{l}\text { Politica y } \\
\text { Gestión } \\
\text { Educacional - } \\
\text { REDIB }\end{array}$ \\
\hline
\end{tabular}




\begin{tabular}{|c|c|c|c|c|}
\hline & & & $\begin{array}{l}\text { dificultades de la } \\
\text { alfabetización digital y } \\
\text { apropiación de los } \\
\text { recursos tecnológicos } \\
\text { para la promoción de } \\
\text { igualdad y } \\
\text { participación social. }\end{array}$ & \\
\hline $\begin{array}{l}\text { Roja } \\
\text { s-Rojas } \\
\text { et al. } \\
(2017)\end{array}$ & $\begin{array}{l}\text { Descripti } \\
\text { vo } \\
\text { explorato } \\
\text { rio con } \\
\text { selección } \\
\text { aleatoria de } \\
\text { la muestra; } \\
\text { encuestas, } \\
\text { en sistema } \\
\text { braille y } \\
\text { traductor } \\
\text { digital de } \\
\text { lengua de } \\
\text { señas }\end{array}$ & $\begin{array}{l}\quad \text { Personas } \\
\text { jóvenes y } \\
\text { adultas } \\
\text { bachilleres de } \\
\text { los } \\
\text { programas de } \\
\text { la Facultad } \\
\text { de Estudios a } \\
\text { Distancia } \\
\text { (FESAD), } \\
\text { Universi } \\
\text { dad } \\
\text { Pedagógica y } \\
\text { Tecnológica } \\
\text { de Colombia } \\
\text { (UPTC). }\end{array}$ & $\begin{array}{l}\text { Los resultados } \\
\text { presentados muestran } \\
\text { que, del grupo de } \\
\text { bachiller es jóvenes el } \\
\text { 53\% son mujeres; la } \\
\text { edad 57\% entre } 18 \text { y } \\
21 \text { - Del grupo de } \\
\text { adultos } 51 \% \text { son } \\
\text { hombres; } 47 \% \text { tiene } \\
\text { entre los } 22 \text { y } 30 \text { años } \\
\text { y } 65 \% \text { no trabaja. Las } \\
\text { de preferencia para } \\
\text { estudiar son: jóvenes - } \\
28 \% \text { ingenierias, } 21 \% \\
\text { ciencias económicas y } \\
\text { administrativas, } 15 \% \\
\text { artes y } 13 \% \text { ciencias de } \\
\text { la educación. Adultos - } \\
\text { el } 27 \% \text { de la ingeniería, } \\
18 \% \text { ciencias } \\
\text { económicas y } \\
\text { administrativas, } 15 \% \\
\text { ciencias de la } \\
\text { educación. de los } \\
\text { participantes } 85 \% \text { del } \\
\text { joven y el } 71 \% \text { del } \\
\text { adulto son personas }\end{array}$ & $\begin{array}{l}\text { Educación } \\
\text { - REDIB }\end{array}$ \\
\hline
\end{tabular}




\begin{tabular}{|c|c|c|c|c|}
\hline & & & $\begin{array}{l}\text { con discapacidad } \\
\text { auditiva. }\end{array}$ & \\
\hline $\begin{array}{l}\text { Burc } \\
\text { i y } \\
\text { Costa } \\
(2018)\end{array}$ & 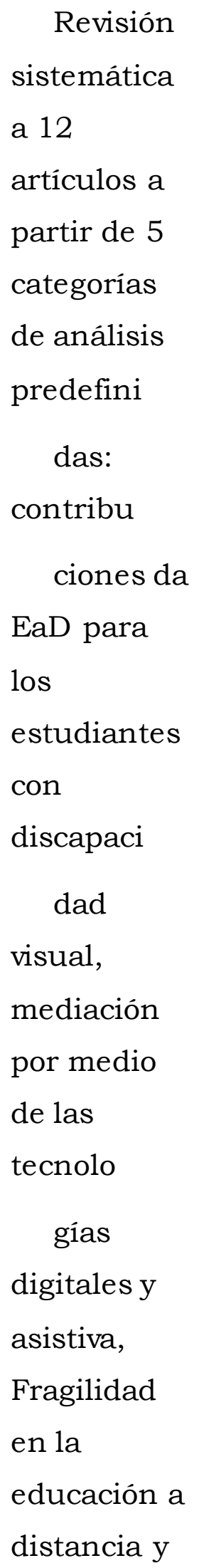 & $\begin{array}{l}\text { Estudiante } \\
\text { s con } \\
\text { discapacidad } \\
\text { visual }\end{array}$ & $\begin{array}{l}\text { Los resultados } \\
\text { indican que dentro de } \\
\text { las estrategias de } \\
\text { tecnología asistiva para } \\
\text { el aprendizaje de los } \\
\text { estudiantes ciegos o } \\
\text { con baja visión existen } \\
\text { diversos recursos. los } \\
\text { estudios analizados } \\
\text { demuestran una } \\
\text { autonomía de los } \\
\text { estudiantes con } \\
\text { discapacidad visual y } \\
\text { de la interacción con } \\
\text { otros, en entornos } \\
\text { virtuales, en que } \\
\text { algunos casos la } \\
\text { discapacidad pasa } \\
\text { desapercibida. }\end{array}$ & $\begin{array}{l}\text { Educación } \\
\text { - REDIB }\end{array}$ \\
\hline
\end{tabular}




\begin{tabular}{|c|c|c|c|c|}
\hline & \begin{tabular}{l} 
el \\
estudiante \\
con \\
discapaci \\
\multicolumn{1}{c}{ dad en } \\
periodo de \\
búsqueda \\
fue de 2000 \\
a 2015 en \\
revistas de \\
revisión por \\
pares con \\
alto nivel de \\
calificación \\
(A2, B1, B2 \\
y B4) en \\
Brasil.
\end{tabular} & & & \\
\hline $\begin{array}{l}\quad \text { Del } \\
\text { Rio et } \\
\text { al } \\
(2018)\end{array}$ & $\begin{array}{l}\text { Estudio } \\
\text { de caso }\end{array}$ & $\begin{array}{l}\quad \text { Centro de } \\
\text { Educación } \\
\text { Especial } \\
\text { Fernando } \\
\text { Arce, } \\
\text { Torrelavega } \\
\text { (Cantabria). }\end{array}$ & \begin{tabular}{l}
\multicolumn{1}{c}{ Los resultados } \\
abordan de una \\
propuesta educativa \\
que supone el \\
reconocimiento y la \\
posibilidad del uso \\
educacional de las \\
personas con \\
discapacidad. El \\
"Proyecto \\
Redconsejos", es una \\
oportunidad al \\
alumnado con \\
discapacidad de \\
participar de un \\
aprendizaje inclusivo.
\end{tabular} & $\begin{array}{l}\text { Educación } \\
\text { Mediática y } \\
\text { TIC - } \\
\text { DIALNET }\end{array}$ \\
\hline
\end{tabular}




\begin{tabular}{|c|c|c|c|c|}
\hline $\begin{array}{l}\text { Med } \\
\text { rano y } \\
\text { Danta } \\
(2018)\end{array}$ & $\begin{array}{l}\text { Sistemati } \\
\text { zación de } \\
\text { dados del } \\
\text { Taller de } \\
\text { Braille } \\
\text { virtual }\end{array}$ & $\begin{array}{l}\quad 10 \\
\text { Docentes de } \\
\text { la ciudad de } \\
\text { Joao } \\
\text { Pessoa/Brasi } \\
\text { 1. }\end{array}$ & $\begin{array}{l}\text { El estudio identificó } \\
2 \text { temas recurrentes en } \\
\text { la comunicación de los } \\
\text { participantes: } 1 \text { ) la } \\
\text { participación del } \\
\text { Braille Virtual como } \\
\text { una herramienta de } \\
\text { accesibilidad } \\
\text { pedagógica e } 2 \text { ) críticas } \\
\text { a esta herramientay } \\
\text { evaluaciones de su } \\
\text { potencial para el } \\
\text { aprendizaje de Braille } \\
\text { por parte de docentes. }\end{array}$ & $\begin{array}{l}\text { Educación } \\
\text { DOAF }\end{array}$ \\
\hline $\begin{array}{l}\quad \text { Bru } \\
\text { no y } \\
\text { Nascim } \\
\text { ento } \\
(2019)\end{array}$ & $\begin{array}{l}\text { Entrevis } \\
\text { tas }\end{array}$ & $\begin{array}{l}10 \\
\text { personas con } \\
\text { discapacidad } \\
\text { visual }\end{array}$ & $\begin{array}{l}\text { La investigación } \\
\text { observa avances en los } \\
\text { estudios culturales } \\
\text { sobre el uso de } \\
\text { recursos y aplicaciones } \\
\text { tecnológicas para el } \\
\text { acceso al } \\
\text { conocimiento, dominio } \\
\text { y uso funcional de las } \\
\text { herramientas de } \\
\text { inclusión digital en la } \\
\text { educación de personas } \\
\text { con discapacidad } \\
\text { visual. }\end{array}$ & $\begin{aligned} & \text { Educación } \\
&- \\
& \text { REDIB/Scielo }\end{aligned}$ \\
\hline
\end{tabular}




\begin{tabular}{|c|c|c|c|c|}
\hline $\begin{array}{l}\quad \text { Pear } \\
\text { son et } \\
\text { al } \\
(2019)\end{array}$ & Encuesta & $\begin{array}{l}\text { Docentes y } \\
\text { estudiantes } \\
\text { con } \\
\text { discapacidad }\end{array}$ & $\begin{array}{l}\text { Los resultados } \\
\text { evidencian desafios } \\
\text { que enfrentan los } \\
\text { estudiantes con } \\
\text { discapacidad en la } \\
\text { participación en línea } \\
\text { en: la tarea } \\
\text { colaborativa, } \\
\text { actividades prácticas, } \\
\text { presentaciones } \\
\text { compartidas, } \\
\text { recopilación de datos } \\
\text { actividades o debates. }\end{array}$ & $\begin{array}{l}\text { Educación } \\
\text { - } \\
\text { DOAF }\end{array}$ \\
\hline $\begin{array}{l}\quad \text { Alot } \\
\text { aibi } \\
\text { et.al } \\
(2020)\end{array}$ & $\begin{array}{l}\text { Entrevis } \\
\text { tas, } \\
\text { observa } \\
\text { ción, } \\
\text { cuestiona } \\
\text { rios y } \\
\text { registros de } \\
\text { desempe } \\
\text { ño }\end{array}$ & $\begin{array}{l}\quad \text { Personas } \\
\text { con } \\
\text { discapacidad } \\
\text { visual }-7 \\
\text { mujeres con } \\
\text { una edad } \\
\text { promedio de } \\
27 \text { años. }\end{array}$ & $\begin{array}{l}\text { Los resultados } \\
\text { indicaron una mejora } \\
\text { en las habilidades de } \\
\text { programación de las } \\
\text { participantes, que } \\
\text { fueron detectados. } \\
\text { Además, se observaron } \\
\text { un alto interés en la } \\
\text { programación de } \\
\text { aprendizaje y actitudes } \\
\text { positivas hacia la } \\
\text { experiencia. Como } \\
\text { desafios se } \\
\text { evidenciaron, la } \\
\text { dificultad de la } \\
\text { comprensión de } \\
\text { conceptos abstractos, } \\
\text { navegación de código y }\end{array}$ & $\begin{array}{c}\text { Lenguaje y } \\
\text { Tecnología - } \\
\text { DOAF }\end{array}$ \\
\hline
\end{tabular}




\begin{tabular}{|c|c|c|c|c|}
\hline & & & $\begin{array}{l}\text { algunos problemas } \\
\text { técnicos. }\end{array}$ & \\
\hline $\begin{array}{l}\text { Bun } \\
\text { dyk } \\
(2020)\end{array}$ & Encuesta & $\begin{array}{l}\text { Docentes } \\
\text { de la } \\
\text { educación } \\
\text { secundaria } \\
\text { de Ucrania. }\end{array}$ & $\begin{array}{l}\text { El estudio presenta } \\
\text { como resultados la } \\
\text { comprensión de que } \\
\text { las tecnologias brindan } \\
\text { a las personas una } \\
\text { nueva oportunidad de } \\
\text { aprendizaje de manera } \\
\text { efectiva. Además, } \\
\text { establece posibilita la } \\
\text { comunicación a través } \\
\text { de dispositivos, incluso } \\
\text { de forma remota. } \\
\text { como dificultades } \\
\text { encontradas el autor } \\
\text { apunta a barreras de } \\
\text { implementación de las } \\
\text { Tics en la educación } \\
\text { inclusiva de Ucrania, } \\
\text { debido al alto costo de } \\
\text { nivel de alfabetización } \\
\text { las herramientas } \\
\text { tecnológicas, } \\
\text { especialmente las } \\
\text { tecnologias de } \\
\text { asistencia. También } \\
\text { constituyen como } \\
\text { obstáculos: falta de } \\
\text { programa, Falta de } \\
\text { Internet potente; bajo } \\
\text { dital }\end{array}$ & $\begin{array}{l}\text { Educación } \\
\text { DOAF }\end{array}$ \\
\hline
\end{tabular}




\begin{tabular}{|c|c|c|c|c|}
\hline & & & $\begin{array}{l}\text { actitud conservadora } \\
\text { de los docentes hacia } \\
\text { las innovaciones, falta } \\
\text { de la comunicación en } \\
\text { linea con estudiantes } \\
\text { con necesidades } \\
\text { educativas especiales; } \\
\text { la falta de apoyo a } \\
\text { estudiantes tales como } \\
\text { para el uso de TIC } \\
\text { especializadas. }\end{array}$ & \\
\hline $\begin{array}{l}\text { Rodr } \\
\text { iguez et } \\
\text { al } \\
(2020)\end{array}$ & $\begin{array}{l}\text { Cualitati } \\
\text { vo, } \\
\text { revisión } \\
\text { documen } \\
\text { tal y un } \\
\text { estudio de } \\
\text { caso. }\end{array}$ & $\begin{array}{l}\quad \text { Grupo de } \\
\text { alumnos y } \\
\text { alumnas con } \\
\text { diversidad } \\
\text { funcional en } \\
\text { el contexto } \\
\text { universitario, } \\
\text { dentro del } \\
\text { "Programa } \\
\text { Incluye } \\
\text { Inserta para } \\
\text { la Inserción } \\
\text { Socio- } \\
\text { Laboral", } \\
\text { Universidad } \\
\text { de Castilla-La } \\
\text { Mancha }\end{array}$ & $\begin{array}{l}\text { La investigación } \\
\text { observar por un lado } \\
\text { una satisfacción de la } \\
\text { docencia y alumnado } \\
\text { con el programa } \\
\text { analizado. Por otra } \\
\text { parte, se percibe una } \\
\text { necesidad de } \\
\text { adaptación } \\
\text { personalizada a los } \\
\text { distintos ritmos de } \\
\text { aprendizaje en el } \\
\text { proceso de enseñanza- } \\
\text { aprendizaje virtual en } \\
\text { comparación con el } \\
\text { presencial. }\end{array}$ & $\begin{array}{l}\text { Educación } \\
\text { - DIALNET }\end{array}$ \\
\hline $\begin{array}{l}\text { Silve } \\
\text { ira } \\
\text { et.al. } \\
(2020)\end{array}$ & $\begin{array}{l}\text { Cualitativ } \\
\text { o y } \\
\text { exploratorio } \\
\text { a la }\end{array}$ & $\begin{array}{l}\quad \text { Personas } \\
\text { con } \\
\text { Trastorno del } \\
\text { Espectro }\end{array}$ & $\begin{array}{l}\text { El estudio evidencia } \\
\text { una construcción } \\
\text { narrativa en el } \\
\text { desarrollo de juegos }\end{array}$ & $\begin{array}{l}\text { Educación } \\
\text { - REDIB }\end{array}$ \\
\hline
\end{tabular}




\begin{tabular}{|c|c|c|c|c|}
\hline & $\begin{array}{l}\text { tecnología } \\
\text { asistiva de } \\
\text { alfabetiza } \\
\quad \text { cíon } \\
\text { interactivo } \\
\text { para } \\
\text { Autistas } \\
\text { (LIA) }\end{array}$ & $\begin{array}{l}\text { Autista (TEA) } \\
\text { en } \\
\text { modalidad } \\
\text { EaD }\end{array}$ & $\begin{array}{l}\text { para personas con } \\
\text { autismo para la } \\
\text { promoción de la } \\
\text { comunicación. Todavia } \\
\text { se percibe un déficit } \\
\text { cuestión de inclusión } \\
\text { de algunos tipos de } \\
\text { discapacidad, visto que } \\
\text { el desarrollo } \\
\text { tecnológico parece } \\
\text { concentrarse en las } \\
\text { discapacidades } \\
\text { visuales y auditivas. } \\
\text { En el caso de TEA, } \\
\text { se observa pocos } \\
\text { aplicativos móviles } \\
\text { gratuitos destinados al } \\
\text { ambiente virtual de } \\
\text { aprendizaje. }\end{array}$ & \\
\hline $\begin{array}{l}\text { Tucc } \\
\text { i et.al } \\
(2020)\end{array}$ & $\begin{array}{l}\text { Estudio } \\
\text { de caso. }\end{array}$ & $\begin{array}{l}\text { Proyecto } \\
\text { ISPRS } 2018 \\
\text { sobre } \\
\text { "Recursos de } \\
\text { educación y } \\
\text { capacitación } \\
\text { en } \\
\text { fotogrametría } \\
\text { digital" } \\
\text { Escuelas } \\
\text { públicas } \\
\text { brasileñas. }\end{array}$ & $\begin{array}{l}\text { El estudio relata } \\
\text { que la implementación } \\
\text { pedagógica de los } \\
\text { recursos educativos y } \\
\text { la adopción de } \\
\text { enfoques de enseñanza } \\
\text { inteligentes es un paso } \\
\text { fundamental en la } \\
\text { difusión de las técnicas } \\
\text { geomáticas en la } \\
\text { discapacidad. }\end{array}$ & $\begin{array}{l}\text { Educación } \\
\text { e Ingeniería } \\
\text { DOAF }\end{array}$ \\
\hline
\end{tabular}




\section{Discusión y consideraciones finales}

Este estudio pone de manifiesto el tema de la accesibilidad digital en el campo de la educación inclusiva, donde se percibe que la tecnologia juega un expresivo papel a través de los diferentes modelos de aprendizaje en línea. Mientras tanto, prevalece una brecha digital que aleja a las personas con discapacidad de una educación de calidad, sea ella presencial o virtual.

Se considera relevante la temática especialmente en el contexto actual, en que las necesidades de distanciamiento social impuestas a partir de la Pandemia Covid-19, exigen nuevas formas de pensar la inclusión. De esta manera, no se puede dejar de lado las diferentes demandas de las personas con discapacidad en el ámbito educativo.

Desde allí, los estudios presentados en la revisión sistemática enfatizan la necesidad de introducir un contenido de alfabetización y formación educacional/profesional en diferentes escalones del proceso de enseñanza-aprendizaje, sea, para los alumnos de la primaria, secundaria, superior, posgrado, entre otros. Por ende, las investigaciones hablan de los recursos de los Tics, como herramientas que posibilitan una interacción entre los docentes y estudiantes, donde la apropiación del conocimiento solo puede existir a partir de la construcción y diseminación de la información entre todos. Esto es como parte de una interacción, enfocada, en este caso, en la discapacidad. Lo diferentes trabajos revisados, dejan en claro el imperativo de una escuela democrática, con los derechos de igualdad y acceso al conocimiento e información (Omodei et.al, 2016; Arias-Flores et.al, 2017; Bruno y Nacimiento, 2019; Budnyk y Kotyk, 2020).

Por lo tanto, la accesibilidad digital, se describe en una escuela que sean espacio común para todos, independiente de la condición física, 
intelectual, mental entre otras. Se observa una orientación a acciones de educación inclusiva en que el eje sea la convivencia con la diversidady la experiencia en la adquisición del conocimiento pautado en el uso de objetos digitales accesibles, de un modo que pueda contribuir para que el alumno/a permanezca y se apoye en una educación a distancia disponible para todos (Prioste y Raiça, 2017), Burci y Costa, 2018; Alotaibi, et.al, 2020).

Hay que avanzar a una visión de tecnología asistiva in tegrada para una inclusión plena en el ámbito educacional. Adoptar el aprendizaje exclusivamente en línea y a distancia ha requerido una adaptación en la transferencia de conocimientos y el desarrollo de capacidades. Las nuevas herramientas didácticas deben asegurar el mismo nivel de calidad que las habituales y compensar la ausencia de retroalimentación directa. La modernización y actualización de los materiales de apoyo utilizados para el aprendizaje en línea, entre otros, pueden ayudar a los nuevos enfoques de enseñanza al proporcionar herramientas de aprendizaje claras, interesantes y atractivas (Medrano y Dantas, 2018; Pearson et.al., 2019; Rodriguez, 2020; Tucci, et.al.2020).

Por último, esto no significa negar al problema de la falta de acceso digital de las personas con discapacidad en el ámbito educativo, sino situarlo dentro de las realidades que remarcan y caracterizan el modelo social en que las demandas/ofertas de los alumnos con discapacidad deben tener el mismo propósito que de los sin discapacidad. 


\section{Referencias}

Alotaibi, H., S Al-Khalifa, H., \& AlSaeed, D. (2020). Teaching Programming to Students with Vision Impairment: Impact of Tactile Teaching Strategies on Student's Achievements and Perceptions. Sustainability, 12(13), 5320.

Arias-Flores, H. P., Jadán-Guerrero, J., \& Ramos-Galarza, C. (2017). Experiencias y retos del uso de herramientas de asistencia en programas de educación superior: Caso de estudio de un estudiante con discapacidad visual. CienciAmérica, 6(3), 52-57.

Barton, Len (2001). La discapacidad, el control y la política de la posibilidad. Kikiriki. Cooperación educativa 59-6, 6-16.

Bruno, M. M. G., \& Nascimento, R. A. L. D. (2019). Política de Acessibilidade: o que dizem as pessoas com deficiência visual. Educação \& Realidade, 44(1).

Budnyk, O., \& Kotyk, M. (2020). Use of Information and Communication Technologies in the Inclusive Process of Educational Institutions.

Burci, T. V. L., \& Costa, M. L. F. (2018). Inclusão de pessoas com deficiência visual na e ducação a distância. Acta Scientiarum. Education, 40(2), e32212-e32212.

Cabero Almenara, J., \& Ruiz Palmero, J. (2017). Las Tecnologías de la Información y Comunicación para la inclusión: reformulando la brecha digital. Ijeri. International Journal of Edu cational Re search and Innovation, 9, 16-30.

Cardoso, G. O., \& Giraffa, L. M. M. (2019). Educação digital e educação inclusiva. Revista de Educação ANEC, 45(158), 153-177.

Cinquegrani, Mirian (2020). “¿Dónde está lo que falta? Representaciones y miradas acerca de la discapacidad en la escuela a partir de las narrativas de familias en lucha por el derecho a la educación inclusiva en la provincia de Buenos Aires (2006-2017)". Revista Pasado Abierto (en prensa).

de1 Río, M. B., García-Ruiz, R., \& Rodríguez, M. A. P. (2018). La educomunicación como reto para la educación inclusiva. EDMETIC, 7(1), 66-86.

García-Prieto, V. (2016). La alfabetización digital para personas con discapacidad: un enfoque mediático. In Actas del I Congreso Internacional Comunicación y Pensamiento. Comunicracia y desarrollo social (2016), p 1223-1239. Egregius.

Hamburgo, I. y Bucksch, S. (2017). Educación inclusiva e innovación social digital. Revista de investigación Advances in Social Sciences , 4 (5).

Medrado, B. P., \& Dantas, R. (2018). Docência e inclusão: o braille virtual como ferramenta na formação de professores. Linguage m: Estudos e Pesquisas, 22(1).

Naciones Unidas (2006). Convención Internacional sobre los Derechos de las Personas con discapacidad. https://www.un.org/esa/socdev/enable/documents/tccconvs.pdf.

Naciones Unidas (2015). Objetivos de Desarrollo Sostenible. https://www.un.org/sustainable development/es /

Omodei, J. D., de Oliveira, É. T., de Souza, M. B., \& dos Santos, V. L. (2016). Acessibilidade em Objetos de Aprendizagem na EaD: uma Análise em um Curso de Especialização. EaD em Foco, 6(1).

Palomino, M. C. P. (2017). El futuro docente ante el uso de las TIC para la educación inclusiva. Digital Education Review, (31), 131-148. 
Pearson, V., Lister, K., McPherson, E., Gallen, AM, Davies, G., Colwell, C., Bradshaw, K. \& Collins, T. (2019). Incorporación y mantenimiento de prácticas inclusivas para apoyar a los estudiantes discapacitados en el aprendizaje combinado y en línea. Revista de medios interactivos en educación, 1.

Pletsch, M. D., de Oliveira, M. C. P., \& Colacique, R. C. (2020). Apresentação-inclusão digital e acessibilidade: desafios da educação contemporânea. Revista Docência e Cibercultura, 4(1), 13-23.

Prioste, C., \& Raiça, D. (2017). Inclusão digital e os principais desafios educacionais brasileiros. Revista on line de Política e Gestão Educacional, 860-880.

Rocha, J. S., da Hora Correia, P. C., \& Santos, J. Z. (2021). Jogos digitais e suas possibilidades na/para educação inclusiva. Revista Pedagógica, 23, 1-25.

Rodriguez, Y. G., Calvo, S. M., \& Martín, V. R. (2020). Abordaje de experiencias educativas digitales ante la crisis del COVID-19 en el contexto universitario con el alumnado de diversidad funcional. Revista de Estilos de Aprendizaje, 13(Especial), 32-42.

Rojas-Rojas, L. M., Arboleda-Toro, N., \& Pinzón-Jaime, L. J. (2018). Caracterización de población con discapacidad visual, auditiva, de habla y motora para su vinculación a programas de pregrado a distancia de una universidad de Colombia. Revista Electrónica Educare, 22(1), 97 124.

Romo, N. M. (2017). Las TIC y los escolares del medio rural, entre la brecha digital y la educación inclusiva. Bordón. Revista de pedagogía, 69(3), 41-56.

Silveira, L. C. G., Luiz, J. M., Guterres, L. X., da Silva Mendes, L. F., \& Ribeiro, L. O. M. (2020). Tecnologias Assistivas no contexto da acessibilidade e mobilidade: possibilidades de inclusão digital de autistas na educação a distância. EmRede-Revista de Educação a Distância, 7(2), 61-73.

Toledo Morales, P., \& Llorente Cejudo, M. D. C. (2016). Formación inicial del profe sorado en el uso de Tecnologías de la Información y la Comunicación (TIC) para la educación del discapacitado. Digital Education Review, 30, 123-134.

Tucci, G., Parisi, E. I., Bonora, V., Fiorini, L., Conti, A., Corongiu, M.,Ortiz-Sanz, P. Santamartín, T.R. Maria, \& Arza-García, M. (2020). Improving Quality and Inclusive Education on Photogrammetry: New Teaching Approaches and Multimedia Supporting Materials. The International Archives of Photogrammetry, Remote Sensing and Spatial Information Sciences, 43, 257-264.

Zaduski, J. C. D., Lima, A. V. I., \& Junior, K. S. (2019). Ecossistemas da aprendizagem na era digital: considerações sobre uma formação para professores na perspectiva da educação inclusiva. Revista Diálogo Educacional, 19(60), 269-287.

Zapata, S. A. (2018). Lineamiento sobre tecnologias de comunicación para educación inclusiva en universidades públicas. Edmetic, 7(1), 124-150. 